\title{
Editorial
}

\section{Update on Human Papillomavirus (HPV) Infection}

The papillomavirus are a group of viruses which are grouped together due to their tumourigenicity and the homogeneity of their DNA which infect vertebrates. Thanks to the use of DNA sequencing, approximately 200 strains of human papillomavirus (HPV) have been identified to date with each one showing a particular tropism for specific anatomical sites with skin, mucous membrane, anal and respiratory tract infections being common. The habitual clinical sign of the cutaneous subtypes are the verrucas with their diverse morphologies: verruca vulgar, verruca plana, verruca plantar, anogenital verrucas or condylomas acuminata and Levandowsky-Lutz verruciform epidermodysplasia. Moreover, we know that approximately 40 of these papillomavirus infect the epithelium of the genital tract. The appearance of these localized lesions at this level varies from forms which are traditionally considered to be benign, such as condylomas acuminata, to precursory lesions which often develop into genital cancer.

Studies on the natural history of HPV infection have revealed that a great number of young women are infected at ages of greater sexual activity. The majority of these infections spontaneously remit without consequences. Women younger than 35 years old have been observed to be more susceptible to suffer genital infections by oncogenic virus, which generally disappear, than women over 35 years old in whom the lesions often persist, with clinical and morphological changes and with greater risks of progression.

The spectrum of epithelial abnormalities known as precursors or considered pre-invasive which are considered to be progressive are seen in various forms depending on the anatomical areas they affect. These lesions have been named in various ways. Thus, the concept and terminology of pre-malignant alterations of the cervical epithelium has evolved in parallel with the advancement in the knowledge of their biology and natural history. The concept of intraepithelial neoplasia of the cervix, vulva, vagina, anus or penis (CIN, VIN, VaIN, AIN or PIN), which joined these lesions together into three grades has been modified, although this terminology is still used today in histological diagnosis.

Due to the ever increasing knowledge of the role of HPV in these lesions and paying attention to the subtype of HPV that they contain, we may classify them into high grade or low grade intraepithelial squamous lesions. Besides epidemiological studies supported by molecular techniques and liquid cytology have confirmed the causative role of certain strains of HPV in the development of cervical, vulvar, vaginal, anal and penile cancers.

Its relationship to other cancers (non-melanomic skin, subungeal, retinoblastoma) is being closely investigated with surprisingly positive results.

In this monologue we hope to give a clear and concise vision of the various forms of presentation of HPV, of both those produced by low-risk genotypes (LR-HPV) and those by high-risk (HR-HPV), as well as bringing their treatments up to date.

\section{Rodríguez-Cerdeira}

(Guest Editor)

Dermatology Department, CHUVI \& University of Vigo

Hospital do Meixoeiro

36200 Vigo

Spain

E-mails: carmen.rodriguez.cerdeira@sergas.es/aristoteles_cerdeira@yahoo.es

(C) C. Rodríguez-Cerdeira; Licensee Bentham Open.

This is an open access article licensed under the terms of the Creative Commons Attribution Non-Commercial License (http://creativecommons.org/licenses/by$\mathrm{nc} / 3.0 /$ ) which permits unrestricted, non-commercial use, distribution and reproduction in any medium, provided the work is properly cited. 